\title{
QUALITY OF CARE OF ACUTE STROKE PATIENTS IN LITHUANIA: NATIONAL DATA
}

\begin{abstract}
Aleksandras Vilionskis ${ }^{1,2}$, Inga Slautaite ${ }^{1,2}$, Rytis Masiliunas ${ }^{1,3}$, Jevgenija Zeldakova $^{1,3}$, Vaidas Matijosaitis ${ }^{4}$, Antanas Vaitkus $^{4}$, Povilas Beliaziunas ${ }^{4}$, Laura Sinkunaite ${ }^{4}$, Saulius Taroza ${ }^{5}$, Linas Masiliunas ${ }^{6}$, Andreea Grecu ${ }^{7}$, Dalius Jatuzis ${ }^{1,3}$

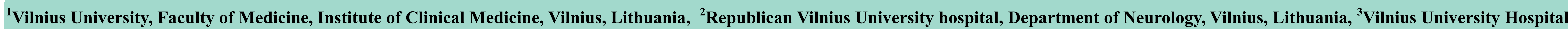

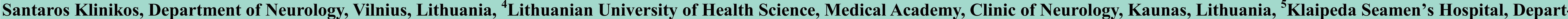

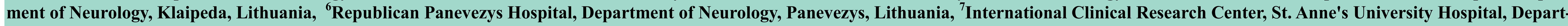
ment of Clinical Neurology, Brno, Czech Republic
\end{abstract}

Background. Quality of care (QOC) of acute stroke patients (ASP) is essential to improve long-term outcomes, decrease mortality and long-term disability. Recent studies have shown that QOC varies across countries and across hospitals in country. The aim of this study was to estimate the QOC of ASP in Lithuania, identify the gaps and suggest ways to improve it.

Patients, methods. In our cross-sectional study all ASP (ischemic stroke, intracerebral hemorrhage and subarachnoid hemorrhage) treated in 5 largest stroke centers in Lithuania during October 2017 were included. The standardized RES-Q questionnaire was used. The questions covered imaging, treatment and secondary prevention topics.

\section{RESULTS}

330 acute stroke patients were included in this study. 305 patients $(92.4 \%)$ had ischemic stroke, 21 patients $(6.4 \%)$ had intracerebral hemorrhage, and 4 patients $(1.2 \%)$ had subarachnoid hemorrhage.

General information

Period of study: March, 2017

Mean age: $69-77$ (across country-73)

Mean NIHSS score on admission: $6-12$ (across country-9)

Number of included patients per centre: RVUH - 92, VUH SK - 59,

LUHSH KK - 47, KSH - 51, RPH - 81

Number of patients received reperfusion therapy:

\begin{tabular}{|llllll|}
\multicolumn{7}{c}{ RVUH } & VUH SK & KUHSH & KSH & RPH \\
\hline IVT & 10 & 10 & 17 & 23 & 6 \\
BT & 1 & 2 & 7 & 2 & 0 \\
MTE & 1 & 4 & 0 & 0 & 2 \\
\hline
\end{tabular}

Figure 3. Dysphagia screening on admission

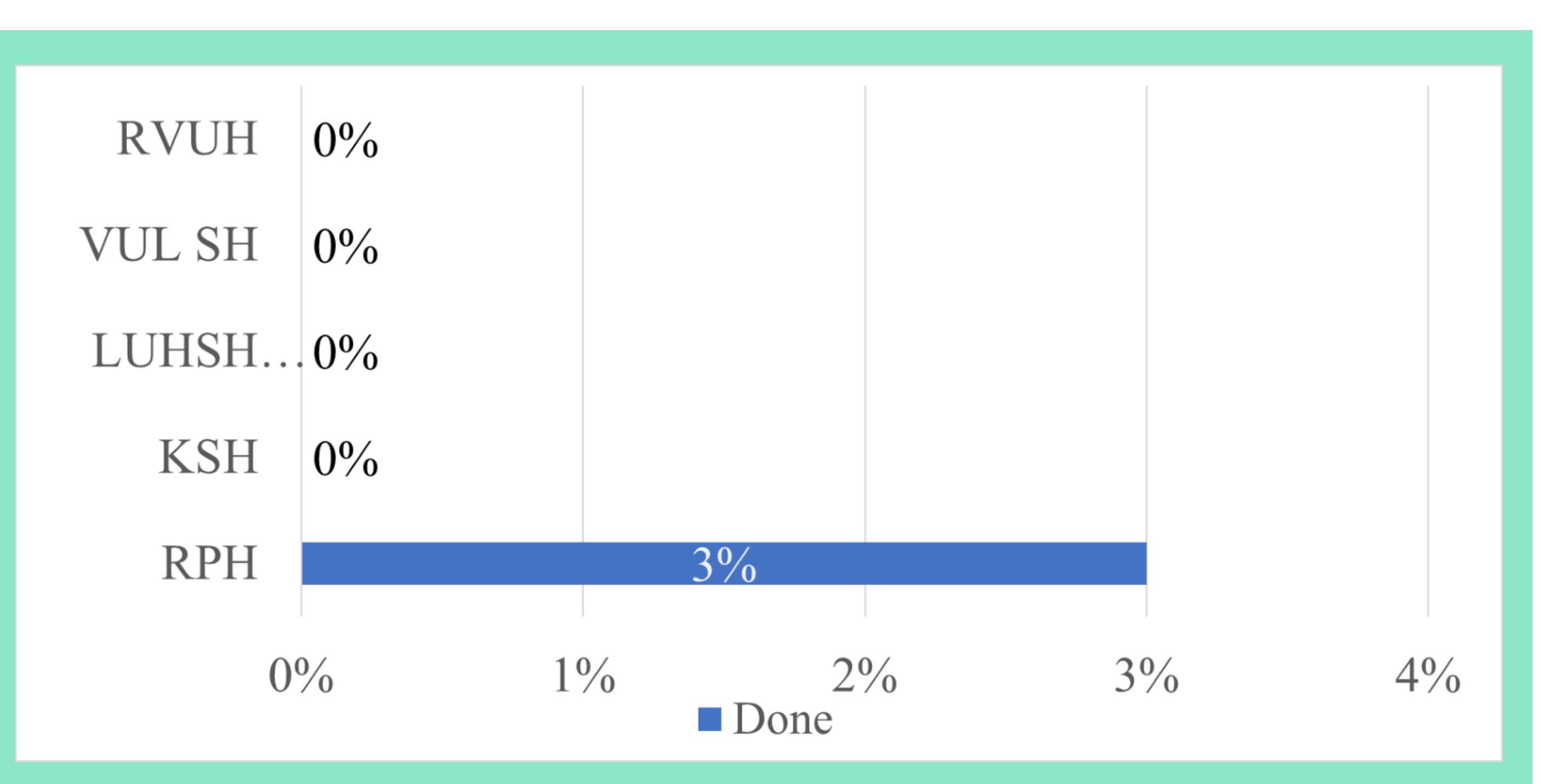

Figure 6. Screening for atrial fibrillation

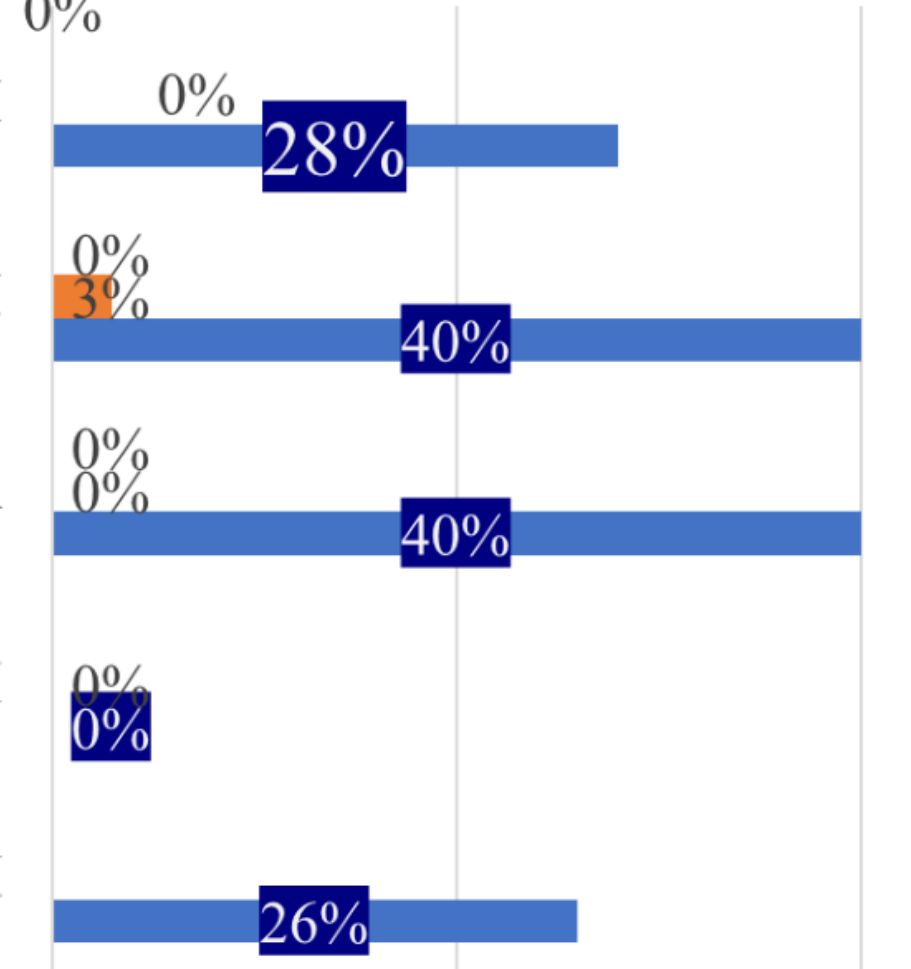

Figure 1. NIHHS score evaluation on admission

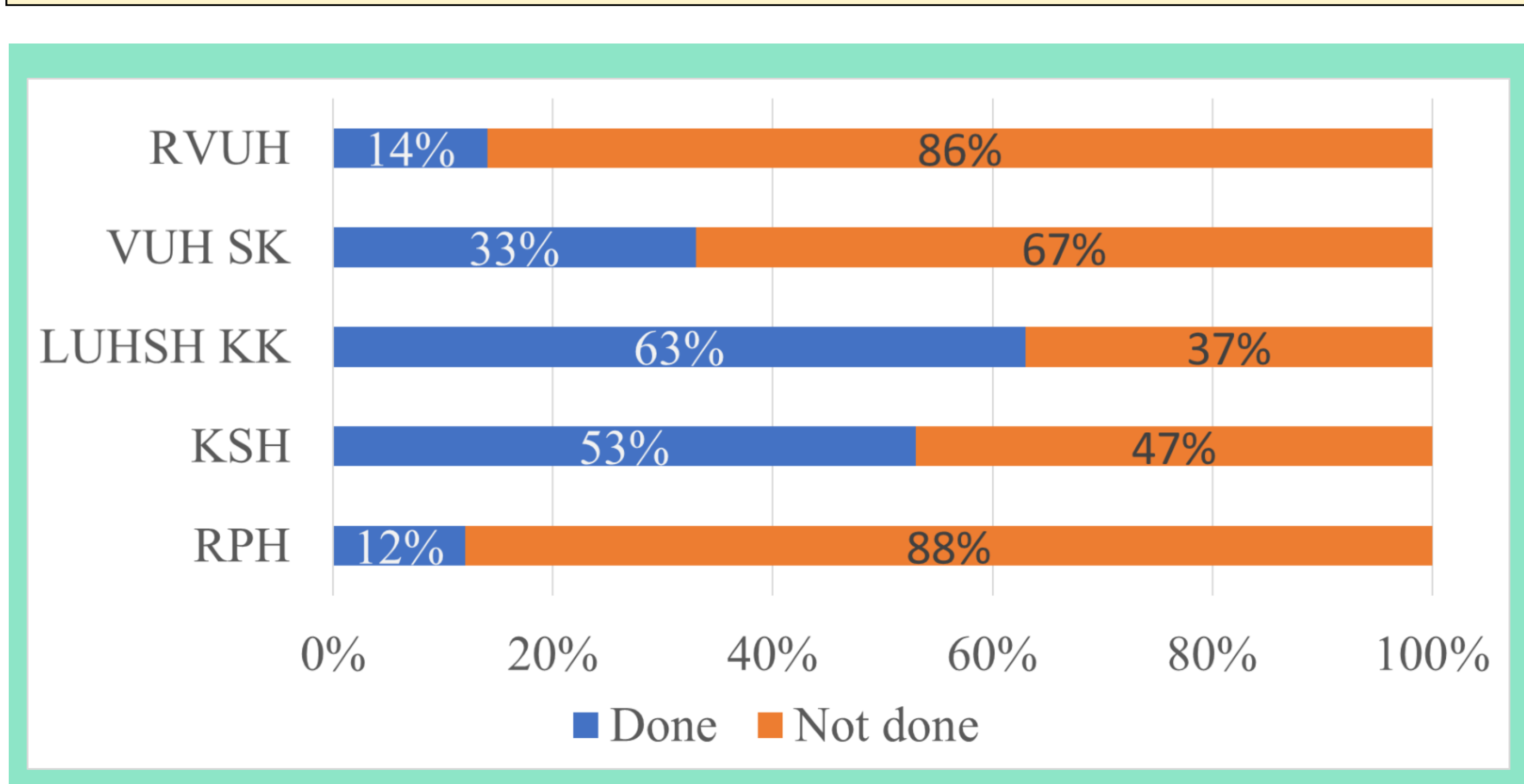

Figure 4. Implementation of reperfusion therapy

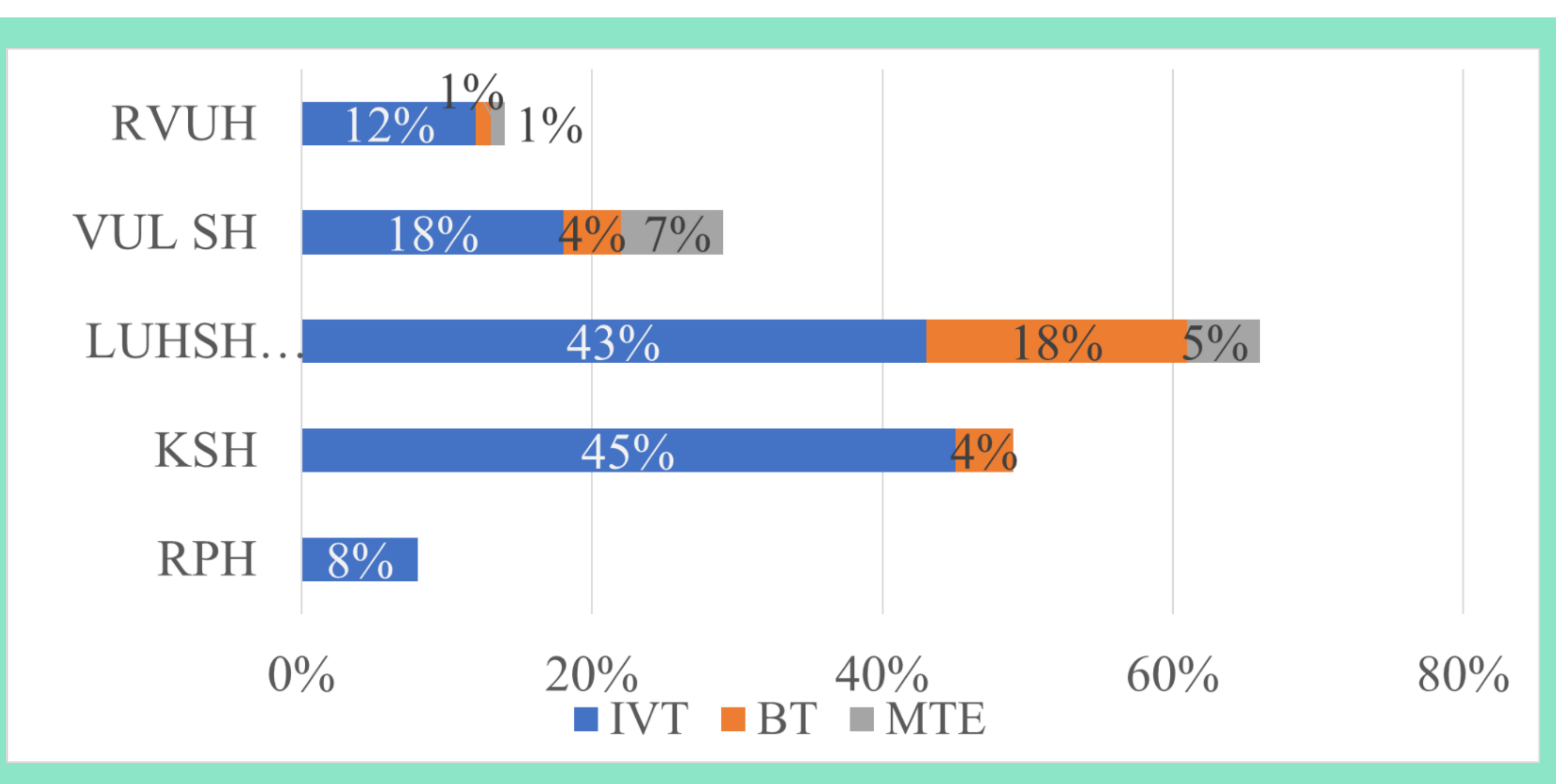

Figure 7. Secondary prevention for non-cardioembolic stroke

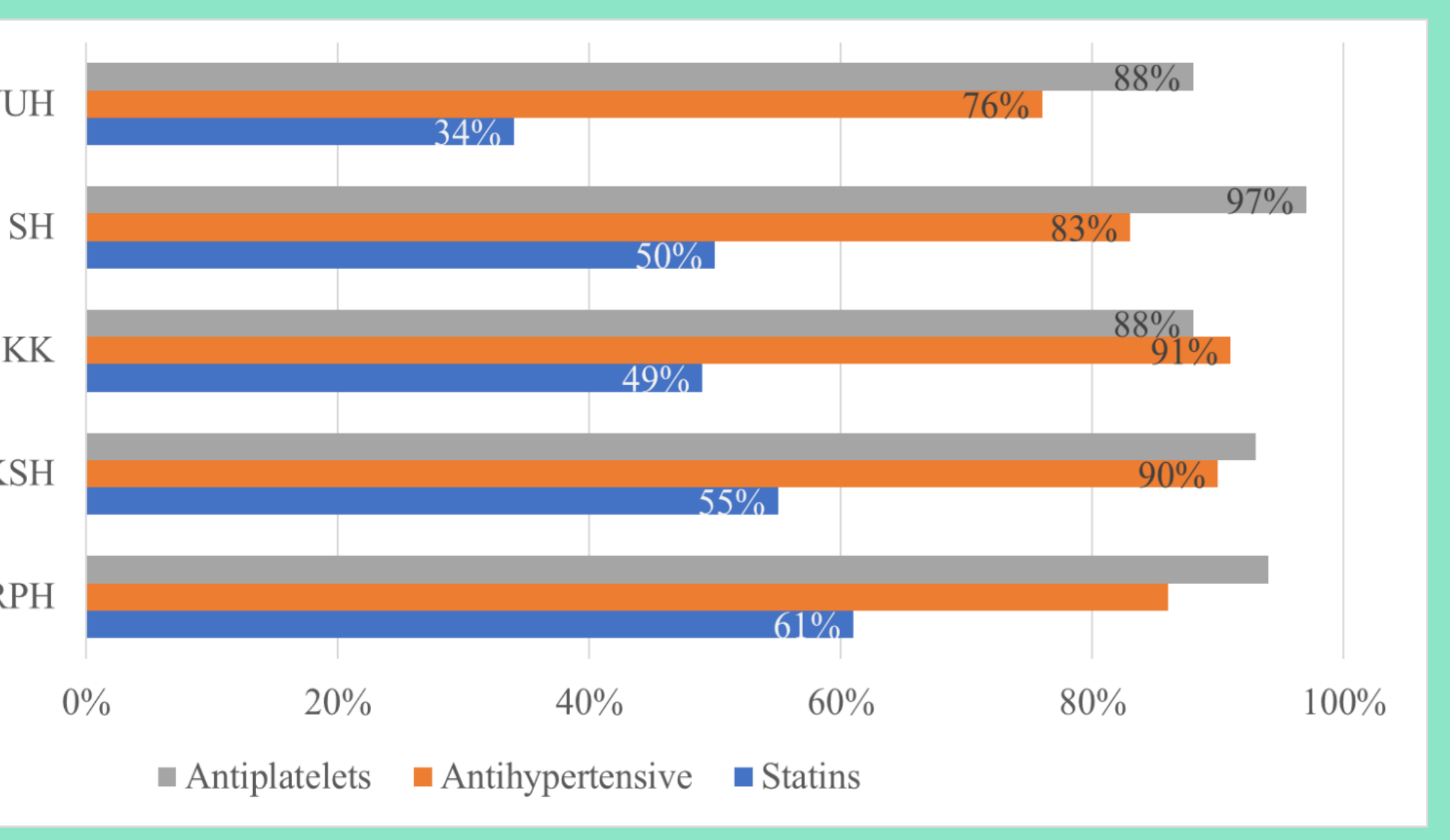

Figure 2. Brain CT on admission

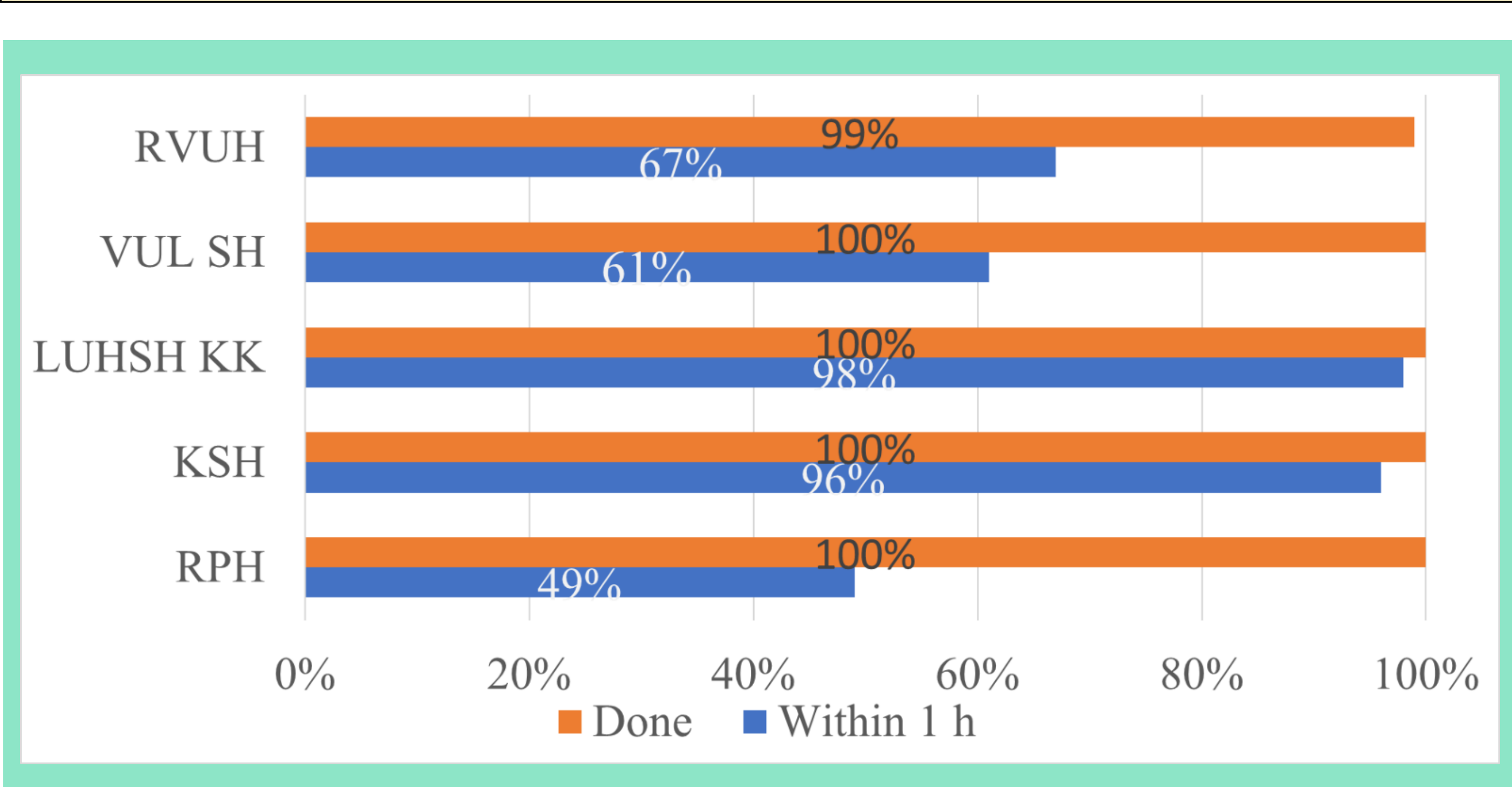

Figure 5. Time delay before procedure

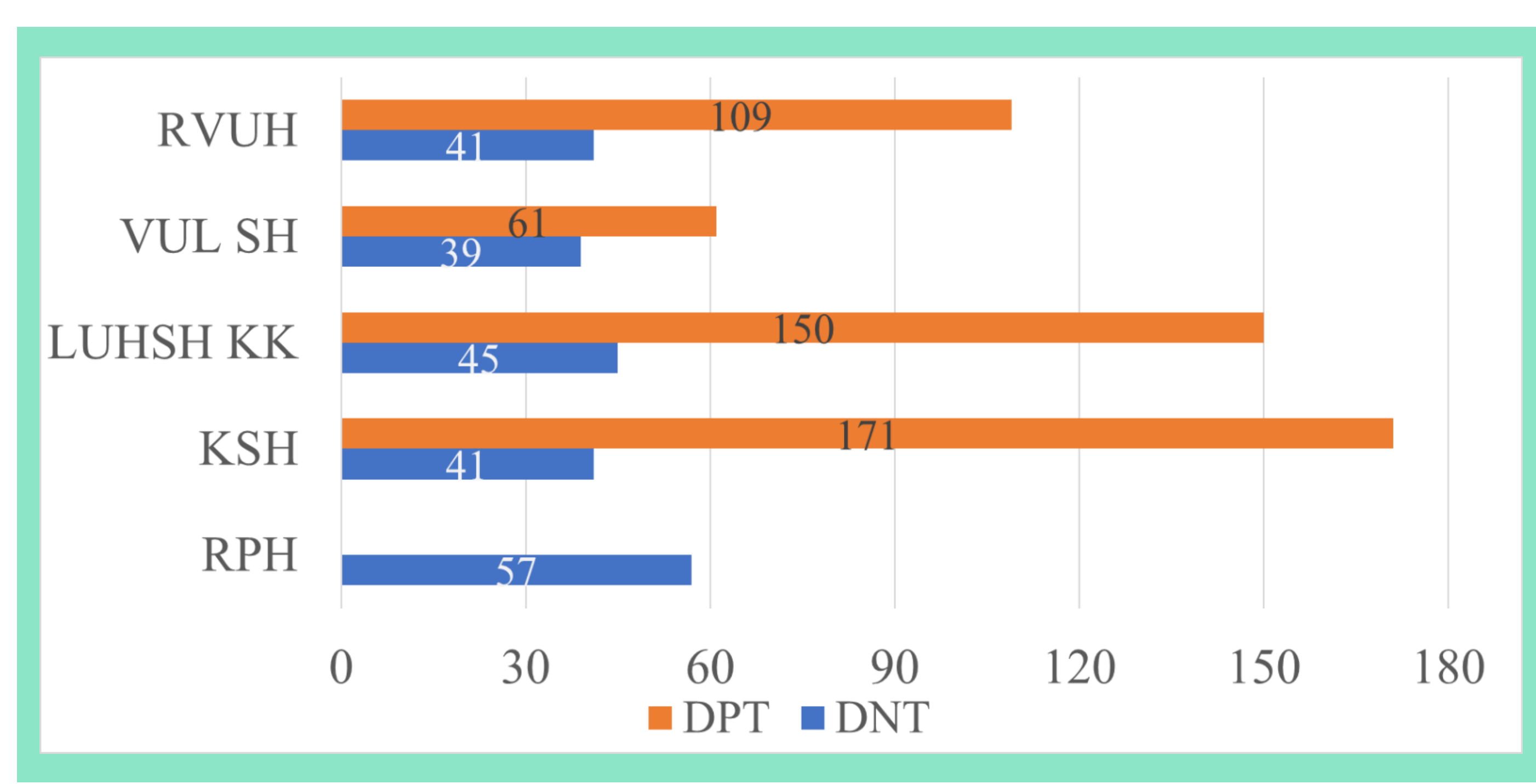

Figure 8. Secondary prevention for cardioembolic stroke

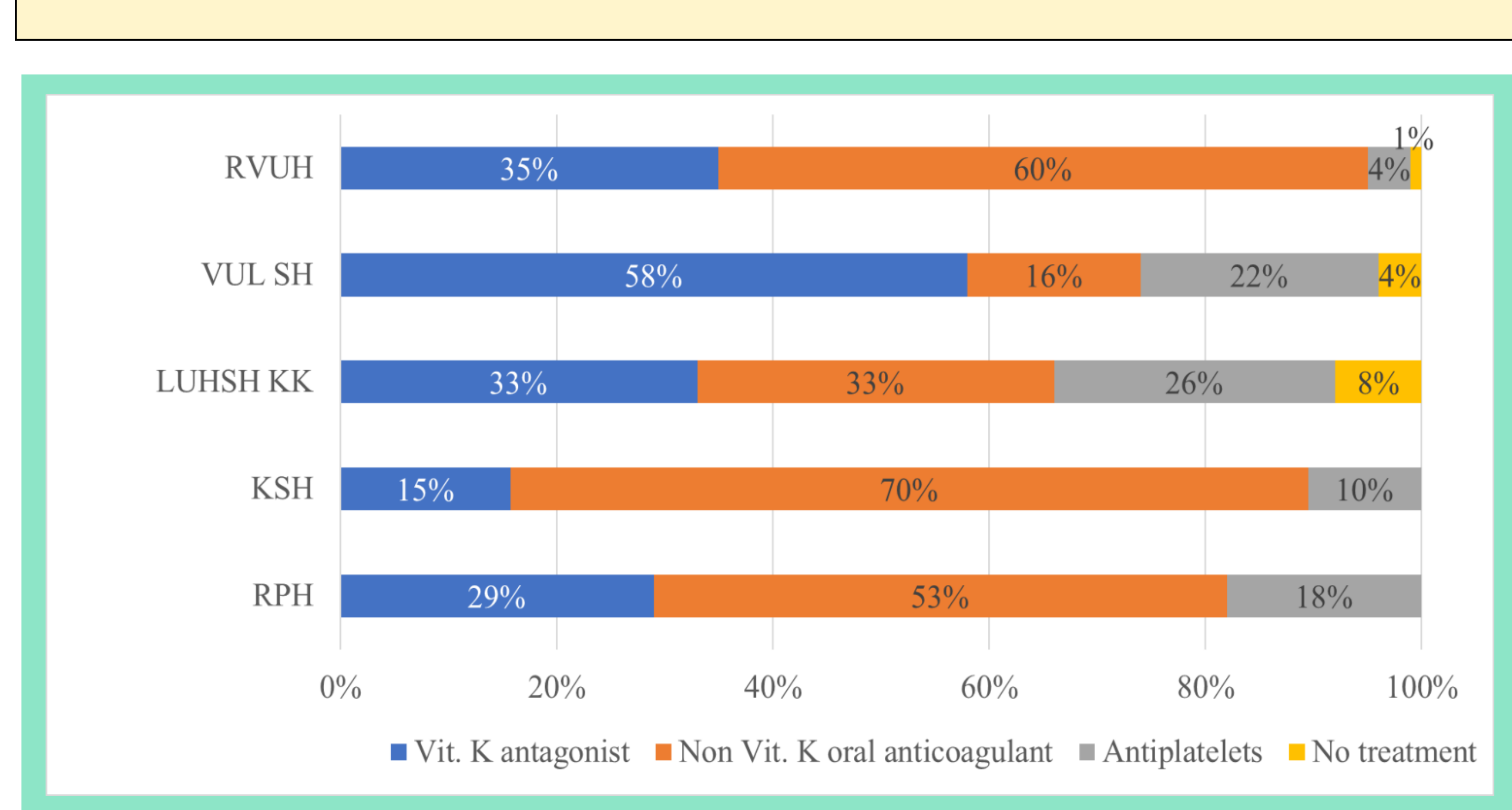

Conclusion. We found that the main gaps in QOC of ASP in Lithuania were insufficient screening for dysphagia and cardiac monitoring for AF. Door-to-needle time should be improved as well. Introduction of a dysphagia protocol and education of hospital staff is needed to improve care for ASP. 